
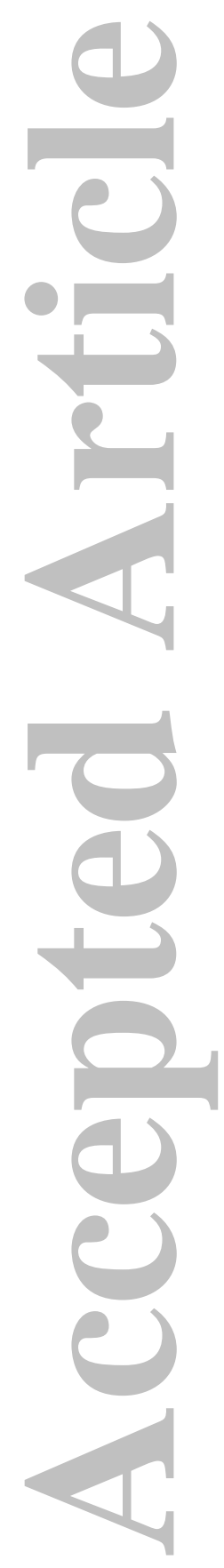

This is the author manuscript accepted for publication and has undergone full peer review but has not been through the copyediting, typesetting, pagination and proofreading process, which may lead to differences between this version and the Version record. Please cite this article as doi:10.1002/ syn.22020. 


\title{
THC alters alters morphology of neurons in medial prefrontal cortex, orbital prefrontal cortex, and nucleus accumbens and alters the ability of later experience to promote structural plasticity
}

\author{
Bryan Kolb ${ }^{1,2}$, Yilin $\mathrm{Li}^{1}$, Terry Robinson ${ }^{3}$, and Linda A. Parker ${ }^{4}$
}

${ }^{1}$ Department of Neuroscience, University of Lethbridge, Lethbridge, Alberta, Canada, T1K 3M4

${ }^{2}$ Canadian Institute for Advanced Research, Toronto, Ontario, Canada

${ }^{3}$ Department of Psychology, University of Michigan, Ann Arbor, Michigan, USA, 48109

${ }^{4}$ Department of Psychology, University of Guelph, Guelph, Ontario, Canada, N1G 2W1

Correspondence to:

Bryan Kolb, Department of Neuroscience, University of Lethbridge, Lethbridge, Alberta, Canada, T1K 3M4. E-mail: kolb@uleth.ca

Acknowledgements

This research was supported by the Natural Sciences and Engineering Research Council of Canada, The Canadian Institutes for Health Research, The Canadian Institute for Advanced Research, and the National Institute on Drug Abuse (USA).

key words: D9-THC; Golgi-Cox; neuroplasticity; enriched environment; dendrite; spines

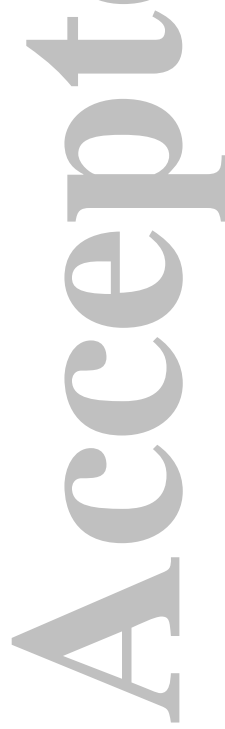




\begin{abstract}
Psychoactive drugs have the ability to alter the morphology of neuronal dendrites and spines and to influence later experience-dependent structural plasticity. If rats are given repeated injections of psychomotor stimulants (amphetamine, cocaine, nicotine) prior to being placed in complex environments, the drug experience interferes with the ability of the environment to increase dendritic arborization and spine density. Repeated exposure to Delta 9-Tetrahydrocannabinol (THC) changes the morphology of dendrites in medial prefrontal cortex (mPFC) and nucleus accumbens (NAcc). In order to determine if drugs other than psychomotor stimulants will also interfere with later experience-dependent structural plasticity we gave Long-Evans rats THC (0.5 $\mathrm{mg} / \mathrm{kg}$ ) or saline for 11 days before placing them in complex environments or standard laboratory caging for 90 days. Brains were subsequently processed for Golgi-Cox staining and analysis of dendritic morphology and spine density $\mathrm{mPFC}$, orbital frontal cortex (OFC), and NAcc. THC altered both dendritic arborization and spine density in all three regions, and, like psychomotor stimulants, THC influenced the effect of later experience in complex environments to shape the structure of neurons in these three regions. We conclude that THC may therefore contribute to persistent behavioral and cognitive deficits associated with prolonged use of the drug.
\end{abstract}

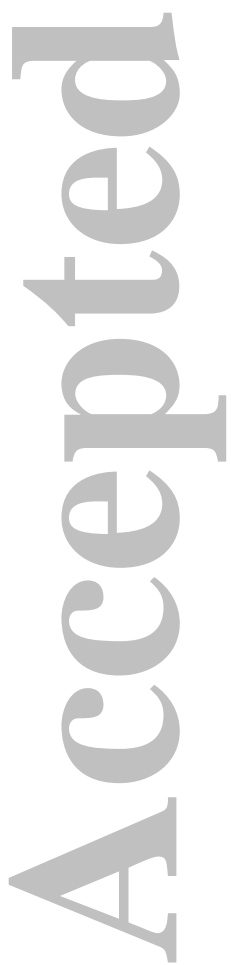




\section{INTRODUCTION}

The evolving views on legalization of marijuana for recreational use in the USA, Canada, and various European countries has led to a growing interest in the long-term effects of cannabis use on the integrity of human brain structures, especially in regions rich in cannabionoid CB1 receptors, including prefrontal cortex, hippocampus, amygdala, striatum, and cerebellum. Although there are now many studies using structural MRIs to evaluate the long-term effects of cannabis use, the results have been inconsistent with early studies showing significant loss of grey and/or white matter (e.g., Battistella et al., 2014; Churchwell et al., 2010) whereas more recent studies have failed to confirm this, possibly because of various comorbid factors (e.g., Weiland et al., 2015), although one study of a large sample of users showed a correlation between cannabis use and white matter integrity and subcortical, but not cortical, morphometry (Orr et al., 2016).

One consistent finding in studies of laboratory animals is that most, if not all, psychoactive drugs alter the structure of neurons in the prefrontal cortex (both medial and orbital areas) and nucleus accumbens, although the nature of the changes vary by class of drug (for a review see Robinson \& Kolb, 2004). Studies of neuronal morphology following exposure to Delta 9-Tetrahydrocannabinol (THC) injections in laboratory animals have been consistent showing alterations in dendritic arborization in prefrontal cortex, striatum (Kolb et al. 2006), hippocampus (Lawston et al., 2000; Scallet et al., 1987), and reductions in a range of measures of cell size in the ventral tegmental dopamine neurons (Behan et al., 2012; Spiga et al., 2010).

In view of the evidence that THC modifies the structure of cerebral neurons the question arises as to whether this might influence subsequent experience-dependent plasticity, such as that associated with learning. A learning experience that is known to produce the largest effects on neuron structure results from housing animals in complex environments (e.g., Siervaag \& Greenough, 1987; van Praag et al., 2000). Furthermore, the administration of psychomotor stimulant drugs to rats (amphetamine, cocaine, nicotine) alters the ability of later experience in a complex environment to change dendritic structure, sometimes occluding such effects (Kolb et al., 2003a; Hamilton \& Kolb, 2005). We therefore asked whether THC might also alter the brain's subsequent response to complex housing. Given that we had previously shown that the effect of THC appeared to be largely limited to medial prefrontal cortex, orbitofrontal cortex, and nucleus accumbens we focused on these structures in the current study. In addition, because we had only examined dendritic branching and length in our earlier study we also wanted to examine the effects of THC on spine density because of the large changes in spine density produced by psychomotor stimulants (e.g., Robinson \& Kolb, 2004). In view of our previous experience both with psychomotor stimulants and complex housing we predicted that the spine changes would be more likely to be found than the changes in dendritic branching and length, both in response to complex housing and THC. We also predicted that like psychomotor stimulants, THC would alter the morphological changes related to complex housing. Both predictions were confirmed.

\section{MATERIALS AND METHODS Animals}

Twenty-four male Long-Evans rats that were born in house from stock originally obtained from 
Quebec Charles-Rivers Laboratory and weighing between 250 and $300 \mathrm{~g}$ at the beginning of the experiment. After weaning the animals lived in pairs in Plexiglas shoebox cages ( 39 X 57 X 21 $\mathrm{cm}$ with corn-cob bedding covering the floor) having ad libitum access to food and water for the duration of the experiment. The colony room was maintained at $21^{\circ} \mathrm{C}$ on a $12 / 12$ light dark schedule, with lights on at 7:30 AM. All experimental procedures were approved by the University of Lethbridge animal welfare committee.

\section{Drugs}

D9-THC (provided by NIDA) was prepared in a suspension of ethanol:cremophor (Sigma):saline $(1: 1: 18)$ and prepared as a solution of $0.5 \mathrm{mg} / \mathrm{ml}$. The rats received either THC $(0.5 \mathrm{mg} / \mathrm{kg})$ or $0.9 \%$ saline i.p. daily for 11 consecutive days. The dose of THC was selected because it is within the estimated range than human marijuana users would obtain in recreational use (Gardner and Lowinson, 1991) and this dose produced structural changes in our previous work (Kolb et al, 2006).

\section{Procedure}

When the experiment began the animals were divided into four groups: Saline $(n=6)$, THC $(n=6)$, Saline-complex $(n=6)$ and THC-complex $(n=6)$. All of the animals remained in pairs in the shoebox cages during the drug injection phase of the study. Each day the rats were transported from their housing to a testing room and prior to drug administration they were placed individually in an activity apparatus for $30 \mathrm{~min}$ prior to drug exposure. Following drug injection the activity was monitored for $60 \mathrm{~min}$ and the rats were returned to their home cages.

The activity system was an AccuScan Instruments Inc., Versamax animal activity monitoring system $\odot$ (open field). The activity apparatus consisted of a clear plexi-glass cage measuring $42 \mathrm{~cm} \times 42 \mathrm{~cm} \times 30 \mathrm{~cm}$ with a removable plexi-glass lid. Each cage was equipped with horizontal and vertical sensors (infra-red beams) that monitored activity in selected intervals for a total of 60 minutes. Recorded measures of horizontal activity (number of beam breaks on the lower bar in each sample period), and total distance (path traveled in centimeters) were retrieved and used for analysis. Once the testing period was complete, the VersaDat function scanned the files for errors to verify the integrity of data collected. The files were then converted for import to an Excel spread sheet.

On the day following the last injection the complex-housed animals were transferred for 90 days to complex environments that consisted of a large $(61$ X 122 X $183 \mathrm{~cm})$ structure. The sides and front of the condo were made of heavy wire mesh and the back vertical wall was galvanized steel. Three platforms were attached to the back wall at various heights with wooden ramps that provided access to each level. The floor was covered with corn-cob bedding. Objects, which included plastic 'toys', paper, cardboard boxes, and PVC pipe, were strewn over the floor and the platforms to encourage exploration. The complex environments were cleaned weekly and the objects replaced each time. As with the lab-caged rats, the complex-housed rats were given ad libitum food and water. The complex environments were in the same animal room as the shoebox cages.

Following the complex housing, the rats were administered an overdose of sodium pentobarbital and perfused intracardially with $0.9 \%$ saline. The brains were removed and placed in $20 \mathrm{ml}$ Golgi-Cox solution and stored in the dark for 14 days. The brains were then placed in a 
$30 \%$ sucrose solution for at least 3 days prior to coronal sectioning (200 um), mounted on glass slides, and stained using a procedures described by Gibb \& Kolb (1998).

The basilar dendrites from layer $\mathrm{V}$ pyramidal neurons were drawn from medial prefrontal cortex (Zilles area Cg3), layer III orbital frontal cortex (Zilles area AID), and medium spiny neurons from the shell of nucleus accumbens (NAcc). The brain regions were first identified at low power $(100 \mathrm{X})$ and five cells from each hemisphere of each region using camera lucida $(250 \mathrm{X})$. In order to be included in the analysis the dendritic trees needed to be intact and not obscured by other neurons, glia, or blood vessels. The dendritic arbor was quantified by counting the number of dendritic branches (indicated by bifrications) using the method of Coleman and Reisen (1968). Dendritic length was estimated using the Sholl (1956) procedure in which an overlay of concentric circles beginning at the cell body and spaced 20 microns apart. The number of intersections of the dendrites and the rings was counted and the length estimated by multiplying by $20 \mathrm{um}$. Spine density was calculated by tracting a length of dendrite (at least $30 \mathrm{um}$ long) at X1000. The exact length of the dendrite was calculated, and the number of spines along the entire length was counted. For cortical pyramidal cells spines were counted on one third-order terminal tip per neuron. For medium spiny NAcc neurons spines were counted on one terminal tip per neuron. No attempt was made to account for the fact that spines on the back side of the dendrites were obscured from view so the spine estimate necessarily underestimates the total spine number. The values for cells in each hemisphere of each rat were averaged, and hemisphere was used as the unit of analysis. The anatomical analyses were performed by someone (Y.L.) blind to the experimental conditions.

Statistical analyses. Statistical analyses were performed using SPSS 21 for Mac. For the behavioral measure, two-way ANOVAs with drug (THC, saline) and test day as variables were performed. The anatomical data for each of the five brain areas were analyzed using three-way ANOVA's with treatment (THC and control), housing, and hemisphere as factors. The data points were the mean of 5 cells per area per hemisphere. However, as hemisphere failed to show significant main effects or interactions with other factors the data were collapsed across this variable and the ANOVA reported as a two-way (Drug and Housing). Posthoc tests were conducted using Tukey's $(\mathrm{p}<.05)$.

\section{RESULTS \\ Locomotor Behavior}

The THC significantly increased activity across the first ten days of drug treatment (see Figure 1). A two-way ANOVA (THC, Test Day) showed a main effect of drug $(\mathrm{F}(11,264)=22.49$, $\mathrm{p}<.0001)$ and test day $(\mathrm{F}(11,264)=18.05, \mathrm{p}<0.001)$ but not the interaction $(\mathrm{F}(11,264)=0.9392$, $\mathrm{p}=0.5031)$.

\section{Anatomical Analysis}

There are three different effects to consider, namely the effect of complex housing, the effect of $\mathrm{THC}$, and the interaction. We consider each region separately. We note that the Golgi staining was good and similar to that seen and illustrated in our previous studies (e.g., Kolb et al., 2003a; 2006).

Cg3. As in our previous studies, dendritic branching, but not length, were affected by complex housing and there was an increase in spine density in the complex-housed rats. THC increased 
dendritic length in both cage and complex reared animals but branching was increased only in the complex-housed group and the THC increased spine density in the complex-housed group but decreased it in the cage-housed group (See Figure 2, Table 1). The statistical analysis for branching showed a significant effect of housing $(\mathrm{F}(1,44)=5.29, \mathrm{p}=.026), \operatorname{THC}(\mathrm{F}(1,44)=5.02$, $\mathrm{p}=.03)$ and the interaction $(\mathrm{F}(1,44)=12.04, \mathrm{p}=.001)$. For length (Sholl line intersections) there was only an effect of THC $(F(1,44)=36.41, \mathrm{p}<.0001)$. Spine density showed a significant effect of housing $(\mathrm{F}(1,44)=43.55, \mathrm{p}<.0001)$ and the interaction $(\mathrm{F}(1,44)=12.63, \mathrm{p}=.0009)$. Thus, exposure to THC changed the effect of complex housing

AID. Complex housing decreased spine density and branching but had no effect on dendritic length. In contrast, THC increased all measures and there were no interactions (see Figure 3, Table1). Analysis of variance on branching showed a significant effect of housing $(\mathrm{F}(1,44)=17.00, \mathrm{p}=.002)$ and $\mathrm{THC}(\mathrm{F}(1,44)=6.42, \mathrm{p}=.04)$, but no interaction. The only statistical effect on length was THC $(\mathrm{F}(1,44)=8.48, \mathrm{p}=.006)$. Spine density showed a significant effect of housing $(\mathrm{F}(1,44)=6.78, \mathrm{p}=.01)$ and $\mathrm{THC}(\mathrm{F}(1,44)=5.31, \mathrm{p}=.03)$, but no interaction.

NAcc. Complex housing increased branching and decreased spine density but did not affect dendritic length. In contrast, THC increased dendritic length and spine density but the branching was only increased in the cage-housed animals (see Figure 4, Table 1). Analysis of variance on branching showed a significant effect of housing $(F(1,44)=17.05, p=.0002)$ and THC $(\mathrm{F}(1,44)=14.56, \mathrm{p}=.0004)$, but no interaction. The only effect on length was THC $(\mathrm{F}(1,44)=36.41, \mathrm{p}=.0001)$. Analysis of spine density showed a significant effect of THC $(\mathrm{F}(1,44)=44.78, \mathrm{p}<.0001)$ and the interaction $(\mathrm{F}(1,44)=6.51, \mathrm{p}=.01)$.

\section{DISCUSSION}

The major results of the current study support the conclusions that 1) THC alters both dendritic arborization and spine density in prefrontal cortex and NAcc; 2) Like psychomotor stimulants, THC interacts with the effects of complex housing on neuronal morphology in prefrontal cortex and NAcc; and, 3) the effects of complex housing vary with cerebral region. We consider each conclusion in turn.

Effects of THC on neuronal morphology

The Kolb et al (2006) study examined the effects of THC on dendritic branching and length in mPFC (Cg3), orbital frontal cortex (AID) parietal cortex (Par1), occipital cortex (Occ1), hippocampus (CA1), striatum, and NAcc. Significant effects were only found in $\mathrm{Cg} 3$ and the shell of NAcc. In the current study we replicated our earlier finding of increased branching in $\mathrm{Cg} 3$ and NAcc and, in addition, showed significant increases in spine density in NAcc but not $\mathrm{Cg} 3$. One different result was that here we found increased branching, length, and spine density in AID. There are three differences between the current study and the Kolb et al (2006) study: 1) the earlier study used Sprague-Dawley rats whereas the current study used Long-Evans; 2) the rats in the current study were born and raised in the University of Lethbridge vivarium whereas in the earlier study the rats were purchased from a breeder and housed at the vivarium at Wilfrid Laurier University; and, 3) in the Kolb et al (2006) there was a withdrawal period of 30 days but in the current study it was 90 days. Therefore, a strain and/or rearing difference may account for the different effects on AID. The 90 day withdrawal period in the current study suggests that the 
THC effects on neuronal morphology are persistent, much like the effects of psychomotor stimulants (e.g., Kolb et al., 2003a).

Overall, both in the current study and in studies of other drug effects on neuronal morphology it appears that the largest changes are seen in spines. This may reflect the fact that spines are the major location of excitatory synapses. The molecular mechanism driving these synaptic changes is uncertain but sensitization to both amphetamine and nicotine is associated with changes in gene expression (Robison \& Nestler, 2011; Mychasiuk et al., 2013).

It is also worth noting that as in our earlier study we found no evidence of behavioral sensitization, which is consistent with other studies reporting that low doses of cannabinoids do not produce behavioral sensitization of locomotor activity (Arnold et al., 1998; Muschamp and Siviy, 2002; Ellgren et al., 2004). Thus, it appears that THC can produce persistent changes in synaptic plasticity, even in the absence of behavioral sensitization. As well, the elevated locomotor activity produced by low doses of THC is consistent with reports that at low doses THC enhances locomotor activity but at high doses it suppresses locomotor activity (e.g. Sañudo-Peña et al, 2000).

\section{THC interacts with the effect of complex housing}

Exposure to THC influences the effect of later experience in complex environments to shape the structure of neurons in prefrontal cortex and NAcc, just as psychomotor stimulants do (amphetamine, cocaine, and nicotine). In fact, it has also been shown that exposure to low doses of nicotine in utero also modifies the effect of complex housing in adulthood (Mychasiuk et al., 2014). Taken together these results suggest that psychoactive drugs can limit or alter the effects of later experiences in modifying neuronal circuits. It is unknown whether similar drug effects would be seen with other types of plastic changes in the brain such as those seen in task learning, long-term potentiation, gonadal hormone manipulations, or cerebral injury. This is an important question because the results of the current study, as well as our earlier ones with psychomotor stimulants, suggest that one long-term consequence of psychoactive drug exposure may be an alteration in behavior and psychological function.

An obvious question that arises is how the drugs interact with plastic changes related to other experiences. There are multiple mechanisms whereby drugs alter the transcriptional potential of genes (for a review see Robison \& Nestler, 2011) and complex housing is associated with changed expression of many genes related to neuronal structure and plasticity (Rampon et al., 2000), increased expression of neurotrophic factors (van Pragg et al., 2000), and FGF-2 reactive astrocytes (Kolb et al., 1998). It thus seems likely that molecular interactions from both types of experience (i.e., drugs and complex housing) could lead to altered synaptic organization, although the details are currently unknown.

Dong \& Nestler (2014) suggested that exposure to psychoactive drugs induces mechanisms of plasticity that normally occur during in reward circuitry during brain development. These re-awakened developmental mechanisms act to form unusually strong and long-lasting maladaptive changes in the brain. Following this logic, we suggest that one of the long-term effects may be an occlusion or modification of other forms of experience-dependent changes, such as what is normally seen in response to complex housing.

\section{Site-dependent changes in the effects of complex housing}

Historically, most studies of the effects of complex housing have been focused on changes in neocortex and hippocampus (e.g., Bennett et al., 1964; Siervaag \& Greenough, 1987; 1988; van 
Praag et al., 2000). In general, although the effects of complex housing appeared larger in posterior than anterior cortex, it has generally been assumed that complex housing induces synaptogenesis across the brain. In the course of studying the effects of experience on prefrontal regions and NAcc we showed that the effects of complex housing are more subtle than elsewhere in the neocortex and, as seen in the current study, the changes in the AID were actually in the opposite direction, namely a pruning of dendritic material and synapses (see also Kolb et al., 2003b). Thus, whereas sensory and motor regions typically show increases in dendritic branching, dendritic length, and spine density changes in mPFC are seen only in an increase in spine density and in OFC there is a reduction in branching and spine density. This opposite effect in the two prefrontal regions has precedence as it is seen in response to both psychomotor stimulants and morphine (Robinson \& Kolb, 2004). We should note that another study (Mychasiuk et al., 2014) placed rats in complex environments for 35 days beginning on postnatal day 60 and although the effects on $\mathrm{Cg} 3$ neurons was similar to the current study, the effects on AID were different with no change in branching or spine density. This inter-study difference appears to reflect the fact that in contrast to $\mathrm{mPFC}$, in which neuronal morphology appears adult by postnatal day 60, neurons in the OFC continue to prune until about postnatal day 90 (Himmler et al., 2017). We might therefore predict that the effects of complex housing will vary by age in OFC (see Kolb et al., 2003c for a parallel effect in parietal cortex).

Finally, there was a reduction in spine density related to complex housing in NAcc in the current study, an effect also seen in Mychasiuk et al. (2014). In contrast, two studies found the opposite result, namely an increase (Kolb et al., 2003a; 2003b). The key difference is that the latter studies were conducted with Sprague-Dawley rats and the studies showing a reduction in spine density used Long-Evans rats. This difference suggests that there could be strain differences in the effects of complex housing, at least in some cerebral regions.

\section{REFERENCES}

Arnold JC, Topple AN, Hunt GE, McGregor IS. 1998. Effects of preexposure and coadministration of the cannabinoid receptor agonist CP 55,940 on behavioral sensitization to cocaine. Eur J Pharmacol 354: 9-16.

Battistella G, Fornari E, Annoni J-M, Chitoui H, Dao K, Fabritius M, Favrat B, Mall J-F, Maeder P, Giroud C. (2014). Long-term effects of cannabis on brain structure. Neuropsychoparmacol 39: 2041-2048.

Behan AT, Hryniewiecka M, O'Tuathaigh CM, Kinsella A, Cannon M, Karayiorgou M, Gogos JA, Waddington JL, Cotter DR. 2012. Chronic adolescent exposure to delta-9tetrahydrocannabinol in COMT mutant mice: impact on indices of dopaminergic, endocannabinoid and GABAergic pathways. Neuropsychopharmacol 37: 1773-1783.

Bennett EL, Diamond MC, Krech D Rosenzweig MR. 1964. Chemical and anatomical plasticity of the brain. Science 146: 610-619.

Churchwell JC, Lopez-Larson M, Yurgelun-Todd DA. 2010. Altered frontal cortical volume and decision making in adolescent cannabis users. Front Psychol 1: 225.

Dong Y, Nestler EJ. 2014. The neural rejuvenation hypothesis of cocaine addiction. Trends Pharmacological Sc 35: 374-383.

Ellgren M, Hurd YL, Franck J. 2004. Amphetamine effects on dopamine levels and behavior following cannabinoid exposure during adolescence. Eur J Pharmcol 497:205-213. 
Gardner EL, Lowinson JH.1991. Marijuana's interaction with brain reward systems: Update. Pharmacol Biochem Behav 40: 571-580

Gibb R Kolb B. 1998. A method for vibratome sectioning of Golgi-Cox stained whole rat brain. J Neurosc Meth 79: 1-4.

Hamilton D, Kolb B. 2005. Nicotine, experience, and brain plasticity. Behav Neurosc 119: 355365.

Himmler BT, Mychasiuk R, Nakahashi A, Himmler SM, Pellis SM, Kolb B. 2017. Juvenile social experience and differential age-related changes in the dendritic morphologies of subareas of the prefrontal cortex in rats. In submission.

Kolb B, Forgie M, Gibb R, Gorny G, Rowntree S. (1998) Age, experience, and the changing brain. Neurosc Biobehav Rev 22: 143-159.

Kolb B, Gorny G, Limebeer CL, Parker LA. 2006. Chronic treatment with Delta-9-

tetrahydrocannabinol alters the structure of neurons in the nucleus accumbens shell and medial prefrontal cortex of rats. Synapse 60: 429-436.

Kolb B, Gorny G, Li Y, Samaha AN, Robinson TE. 2003a. Amphetamine or cocaine limits the ability of later experience to promote structural plasticity in the neocortex and nucleus accumbens. Proc Natl Acad Sci U S A 100: 10523-10528.

Kolb B, Gorny G, Sonderpalm A, Robinson TE. 2003b. Environmental complexity has different effects on the structure of neurons in the prefrontal cortex versus the parietal cortex or nucleus accumbens. Synapse 48: 149-153.

Kolb B, Gibb R, Gorny G. 2003c. Experience-dependent changes in dendritic arbor and spine density in neocortex vary with age and sex. Neurobiol LearnMem: 791, 1-10.

Lawston J, Borella A, Robinson JK, Whitaker-Azmitia PM 2000. Changes in hippocampal morphology following chronic treatment with the synthetic cannabinoid WIN 55, 212-2. Brain Res 877: 407-410.

Muschamp JW, Siviy SM. 2002. Behavioral sensitization to amphetamine follows chronic administration of the CB1 agonist WIN 55, 212-2 in Lewis rats. Pharmacol Biochem Behav 73:835-842.

Mychasiuk R, Muhammad A, Ilnytsky S, Kolb B. 2013. Persistent Gene Expression Changes in NAc, mPFC, and OFC Associated with Previous Nicotine or Amphetamine Exposure. Behav Brain Res 256: 655-651.

Mychasiuk R, Muhammad A, Kolb B. 2014. Environmental Enrichment Alters Structural Plasticity of the Adolescent Brain But Does Not Remediate the Effects of Prenatal Nicotine Exposure. Synapse 68: 293-305.

Orr JM, Paschall CJ, Banich MT. 2016. Recreational marijuana use impacts white matter integrity and subcortical (but not cortical) morphometry. NeuroImage: Clin 12: 47-56.

Rampon C, Jiang CH, Dong H, Tang Y-P, Lockhart DJ, Schultz PG, Tsien JZ, Hu Y. 2000. Effects of environmental enrichment on gene expression in the brain. Proc Natl Acad Sci U S A 97: 12880-12884.

Robinson TE Kolb B. 2004. Structural plasticity associated with drugs of abuse. Neuropharmacol 47 Suppl 1: 33-46.

Sañudo-Peña MC, Romero J, Seale GE, Fernandez-Ruiz JJ, Walker JM. 2000. Activational role of cannabinoids on movement. Eur J Pharmacol 391: 269-274.

Scallet AC, Uemura E, Andrews A, Ali SF, McMillan DE, Paule MG et al 1987. Morphometric studies of the rat hippocampus following chronic delta-9-tetrahydrocannabinol (THC). Brain Res 436: 193-198. 
Sirevaag AM Greenough WT. 1987. Differntial rearing effects on rat visual cortex synapses. III. Neuronal and glial nuclei, boutons, dendrites, and capillaries. Brain Research, 424: 32032.

Sirevaag AM Greenough WT. 1988. A multivariate statistical summary of synaptic plasticity measures in rats exposed to complex, social and individual environments. Brain Research 441:386-92.

Spiga S, Lintas A, Migliore M, Diana M. 2010. Altered architecture and functional consequences of the mesolimbic dopamine system in cannabis dependence. Addiction Biology, 15: 266-276.

Van Praag H, Kempermann G, Gage FH. 2000. Neural consequences of environmental enrichment. Nature Rev Neurosc 1: 191-196.

Weiland BJ, Thayer RE, Depue BE, Sabbineni A, Bryan AD, Hutchison KE. 2015. Daily

marijuana use is not associated with brain morphometric measures in adolescents or adults. J Neurosc 35: 1505-1512.
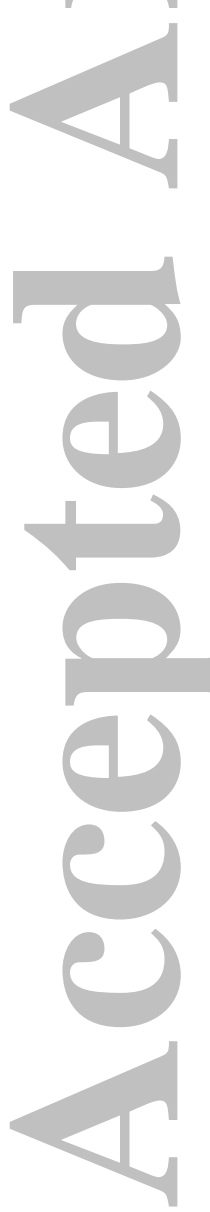
Table 1. Summary of Dendritic Effects

\begin{tabular}{lccc} 
Parameter & Housing & THC & Housing X THC \\
\hline & & & \\
Cg3 branches & $\mathrm{S}$ & $\mathrm{S}$ & $\mathrm{S}$ \\
Cg3 length & $\mathrm{N}$ & $\mathrm{S}$ & $\mathrm{N}$ \\
Cg3 spines & $\mathrm{S}$ & $\mathrm{N}$ & $\mathrm{S}$ \\
& & & \\
AID branches & $\mathrm{S} *$ & $\mathrm{~S}$ & $\mathrm{~N}$ \\
AID length & $\mathrm{N}$ & $\mathrm{S}$ & $\mathrm{N}$ \\
AID spines & $\mathrm{S} *$ & $\mathrm{~S}$ & $\mathrm{~N}$ \\
& & & \\
NAcc branches & $\mathrm{S}$ & $\mathrm{N}$ & $\mathrm{S}$ \\
NAcc length & $\mathrm{N}$ & $\mathrm{S}$ & $\mathrm{N}$ \\
NAcc spines & $\mathrm{S} *$ & $\mathrm{~S}$ & $\mathrm{~S}$ \\
\hline
\end{tabular}

Abbreviations: $S=$ significant increase $\left(\mathrm{p}<.05\right.$ or better); $\mathrm{S}^{*}=$ significant decrease $(\mathrm{p}<.05$ or better); $\mathrm{N}=$ no effect

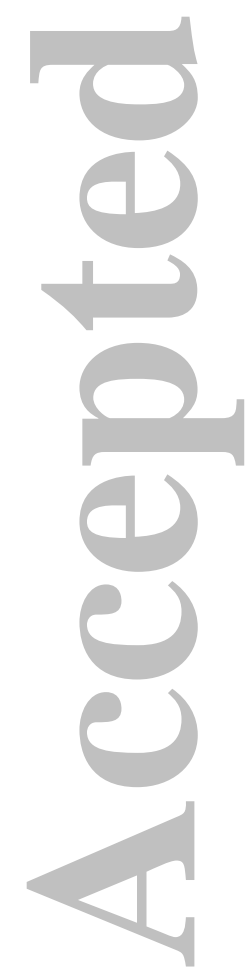




\section{Figure Captions}

Figure 1. Locomotor activity related to THC administration. The THC increased activity but there was no sensitization.

Figure 2. Summary of measures of $\mathrm{Cg} 3$ neurons. There was a significant effect of complex housing on dendritic braching and spine density but not for dendritic length. THC increased dendritic length but THC had housing-specific effects on dendritic branching and spine density (p's $<.05$ or better).

Figure 3. Summary of measures of AID neurons. There was a significant effect of housing and THC on branching, and an effect of THC on dendritic length and on spine density, although only in the complex housed group (p's $<.05$ or better).

Figure 4. Summary of measures of NAcc neurons. THC significantly increased all measures of NAcc neurons. Housing only affected branching and spine density, but the effect on spine density only occurred in THC-treated animals.

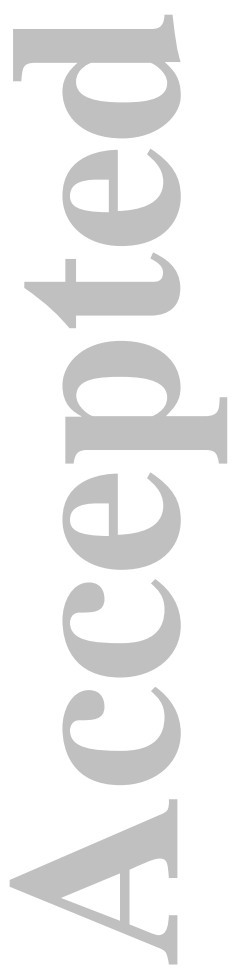


Figure 1.

\section{Locomotor Activity}

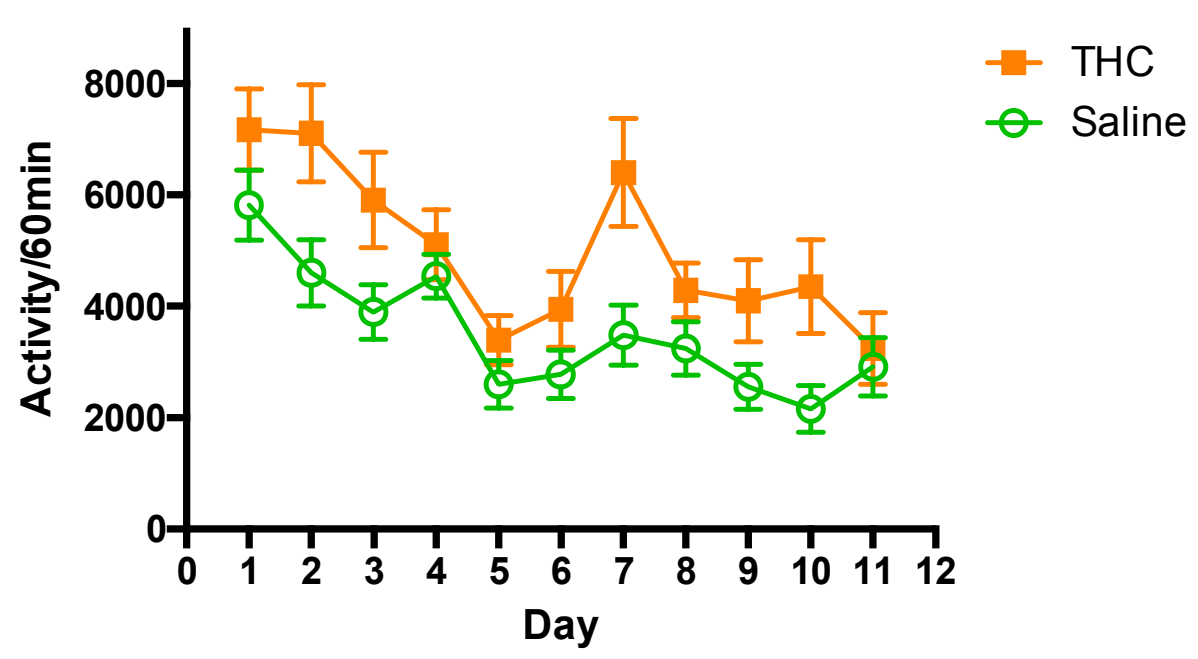

Figure 1. Locomotor activity related to THC administration. The THC increased activity but there was no sensitization. 
Figure 2. Summary of measures of $\mathrm{Cg} 3$ neurons
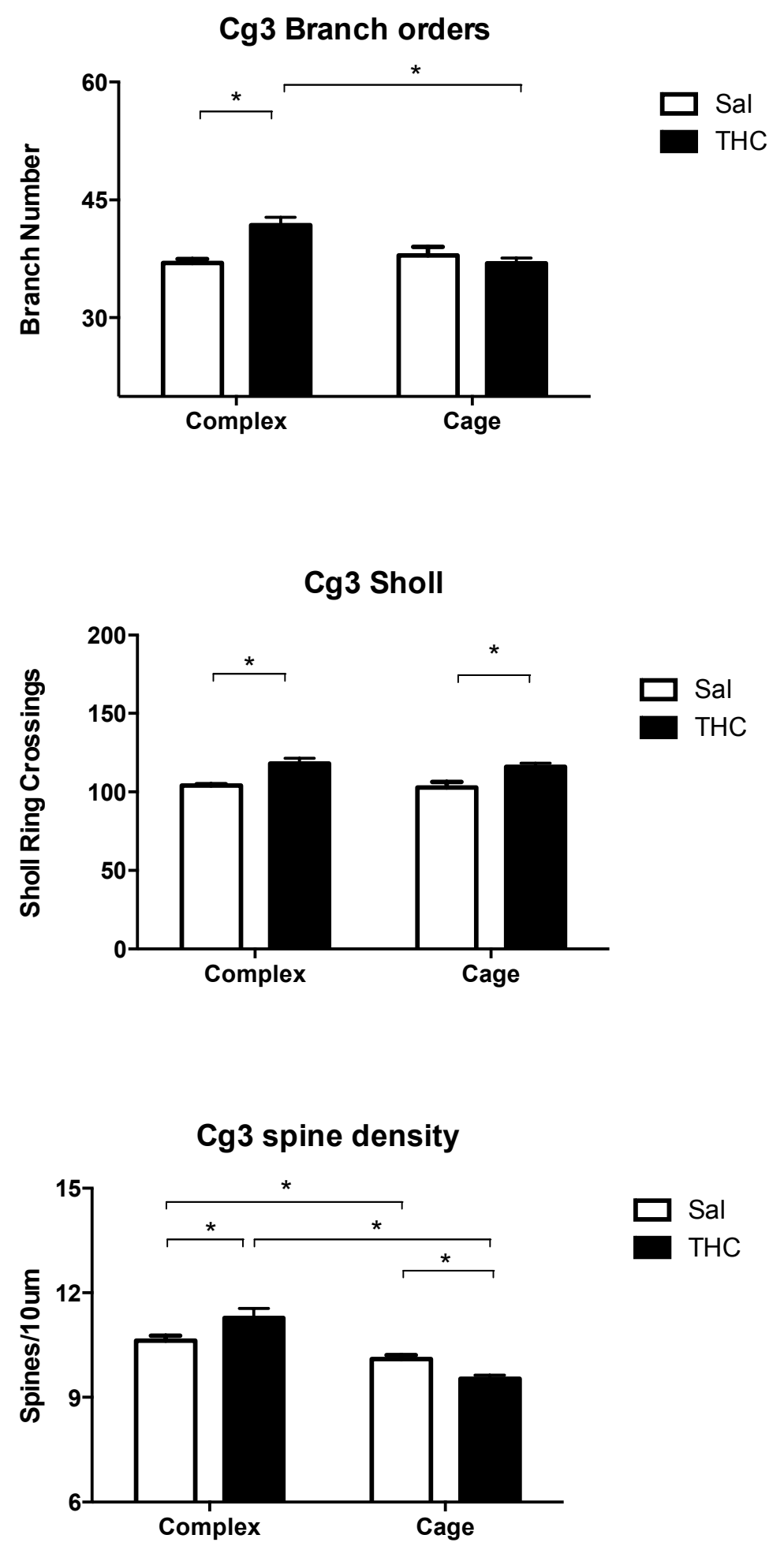
Figure 3. Summary of measures of AID neurons
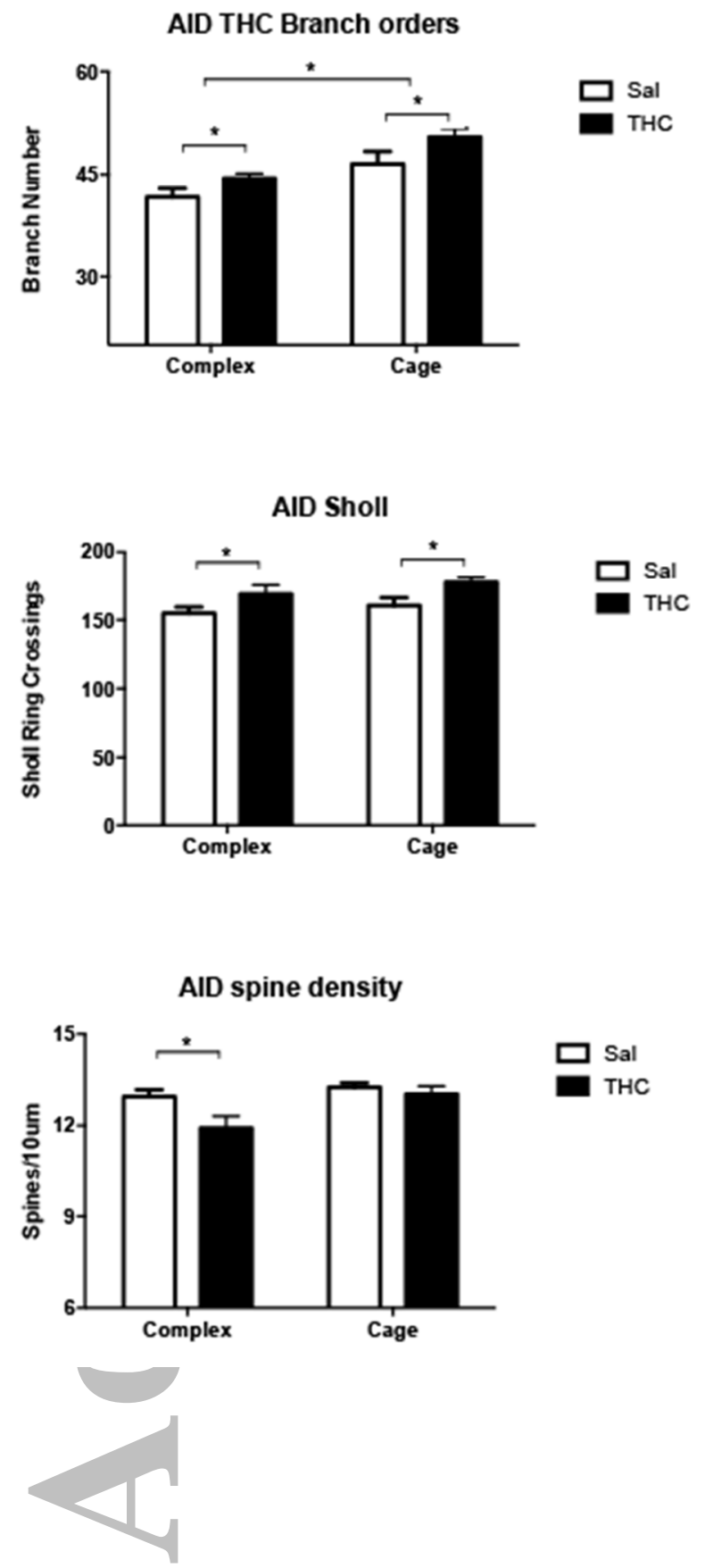
Figure 4. Summary of measures of NAcc neurons
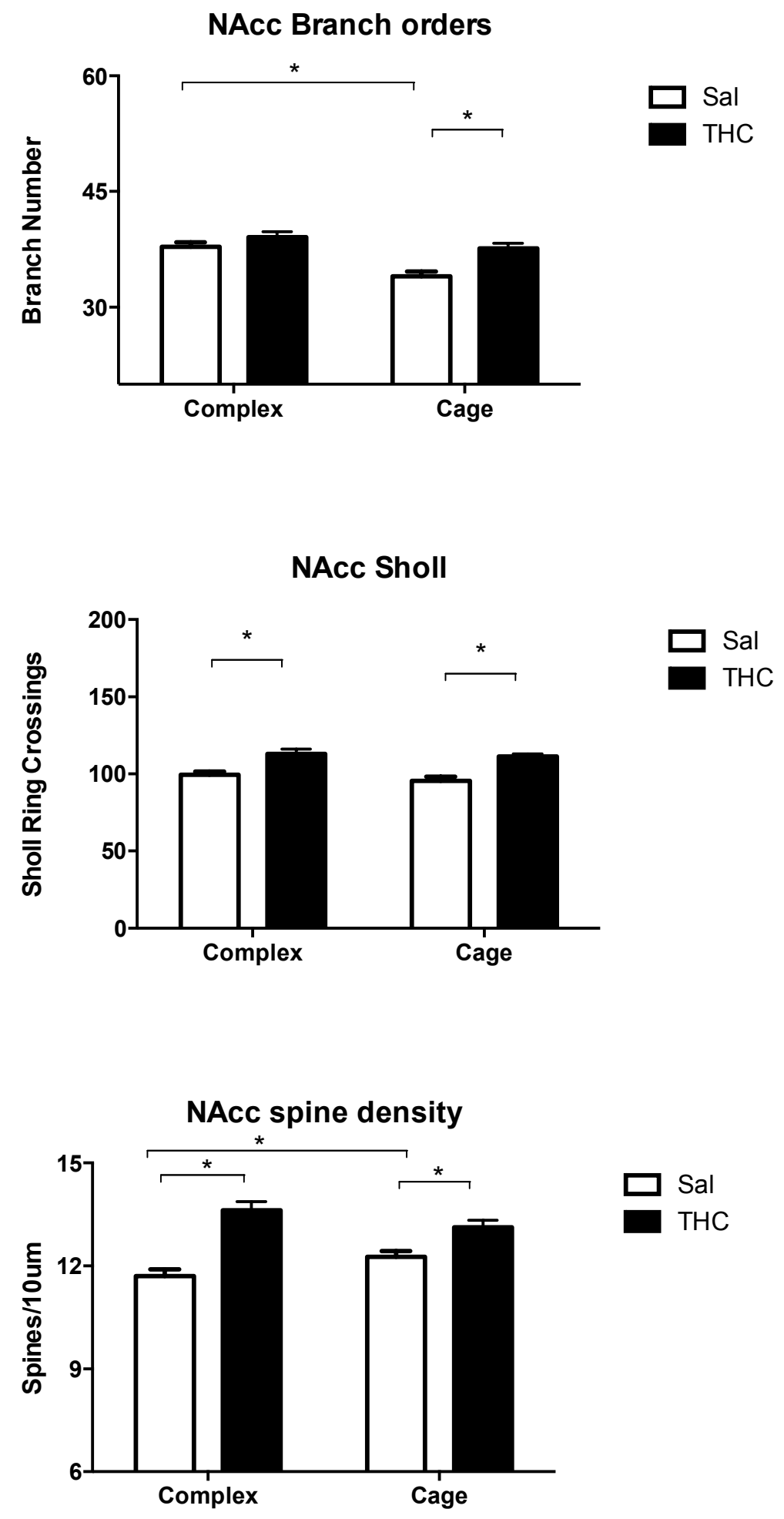
Table 1. Summary of Dendritic Effects

\begin{tabular}{lccc} 
Parameter & Housing & THC & Housing X THC \\
\hline Cg3 branches & S & S & S \\
Cg3 length & $\mathrm{N}$ & $\mathrm{S}$ & $\mathrm{N}$ \\
Cg3 spines & $\mathrm{S}$ & $\mathrm{N}$ & $\mathrm{S}$ \\
AID branches & $\mathrm{S}^{*}$ & $\mathrm{~S}$ & $\mathrm{~N}$ \\
AID length & $\mathrm{N}$ & $\mathrm{S}$ & $\mathrm{N}$ \\
AID spines & $\mathrm{S}^{*}$ & $\mathrm{~S}$ & $\mathrm{~N}$ \\
NAcc branches & $\mathrm{S}$ & $\mathrm{N}$ & $\mathrm{S}$ \\
NAcc length & $\mathrm{N}$ & $\mathrm{S}$ & $\mathrm{N}$ \\
NAcc spines & $\mathrm{S}$ & $\mathrm{S}$ & $\mathrm{S}$ \\
\hline
\end{tabular}

Abbreviations: $\mathrm{S}=$ significant increase; $\mathrm{S}^{*}=$ significant decrease; $\mathrm{N}=$ no effect

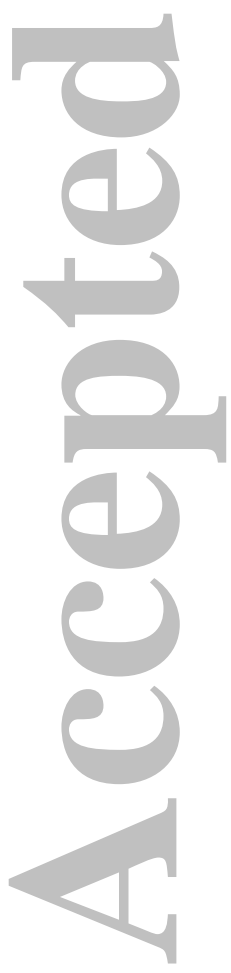




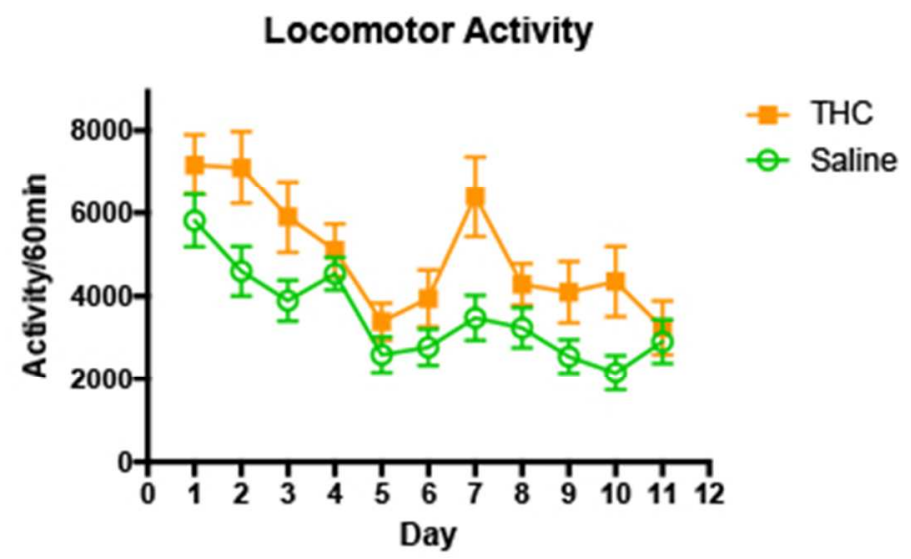

Figure 1. Locomotor activity related to THC administration. The THC increased activity but there was no sensitization. 

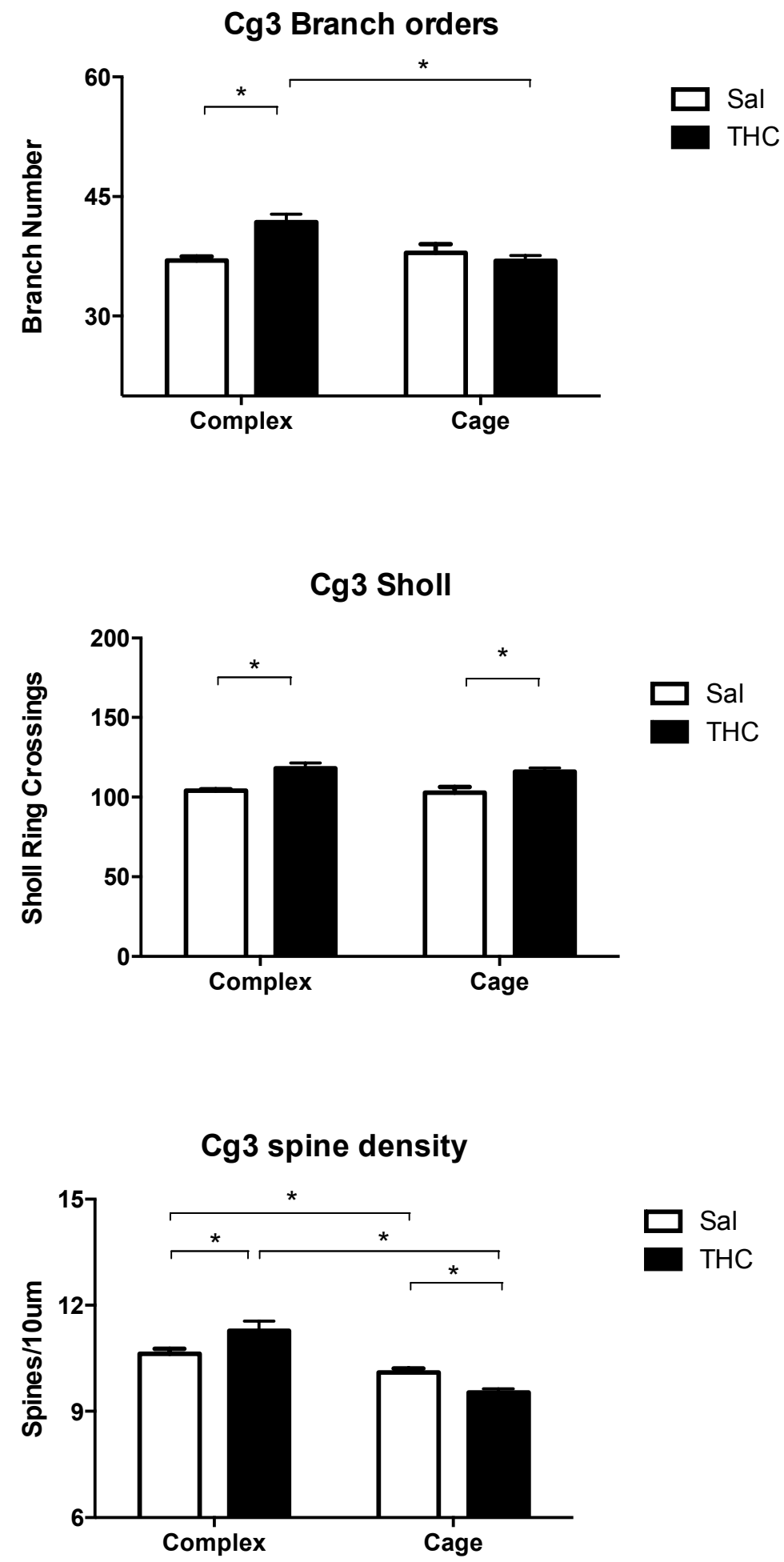

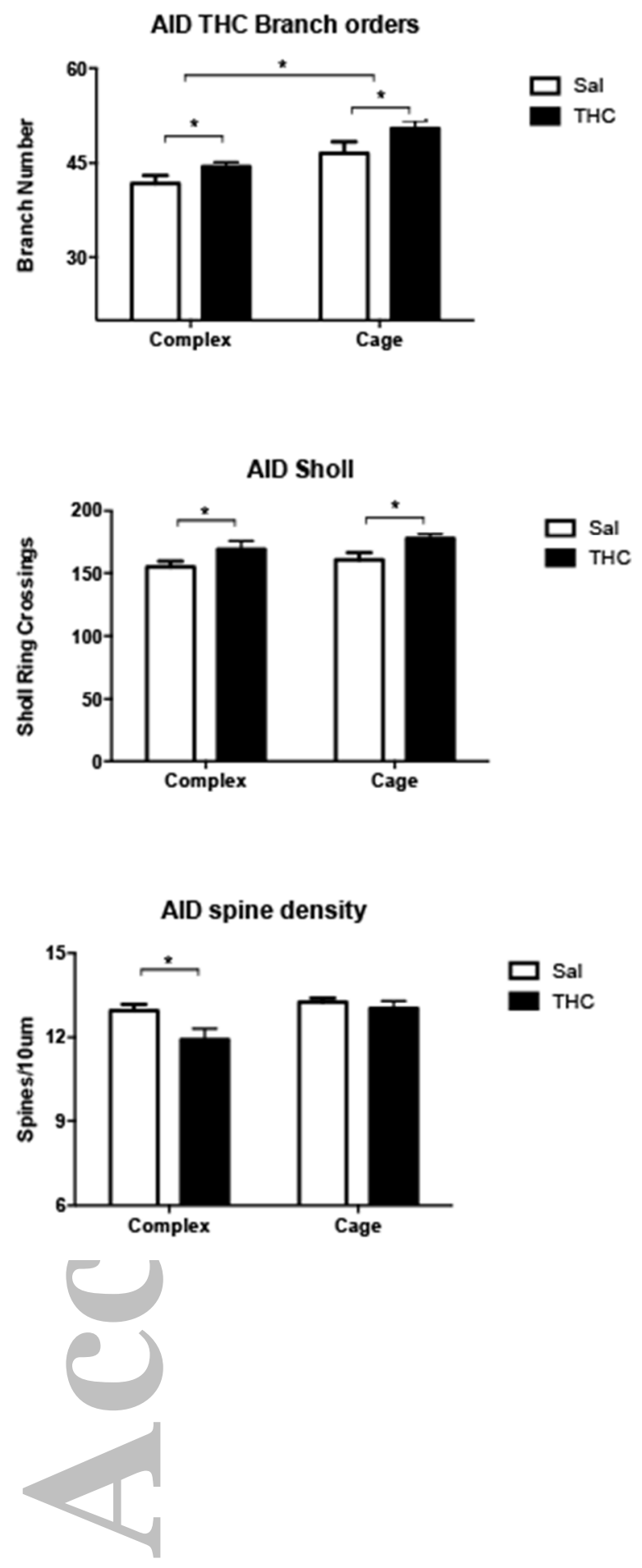

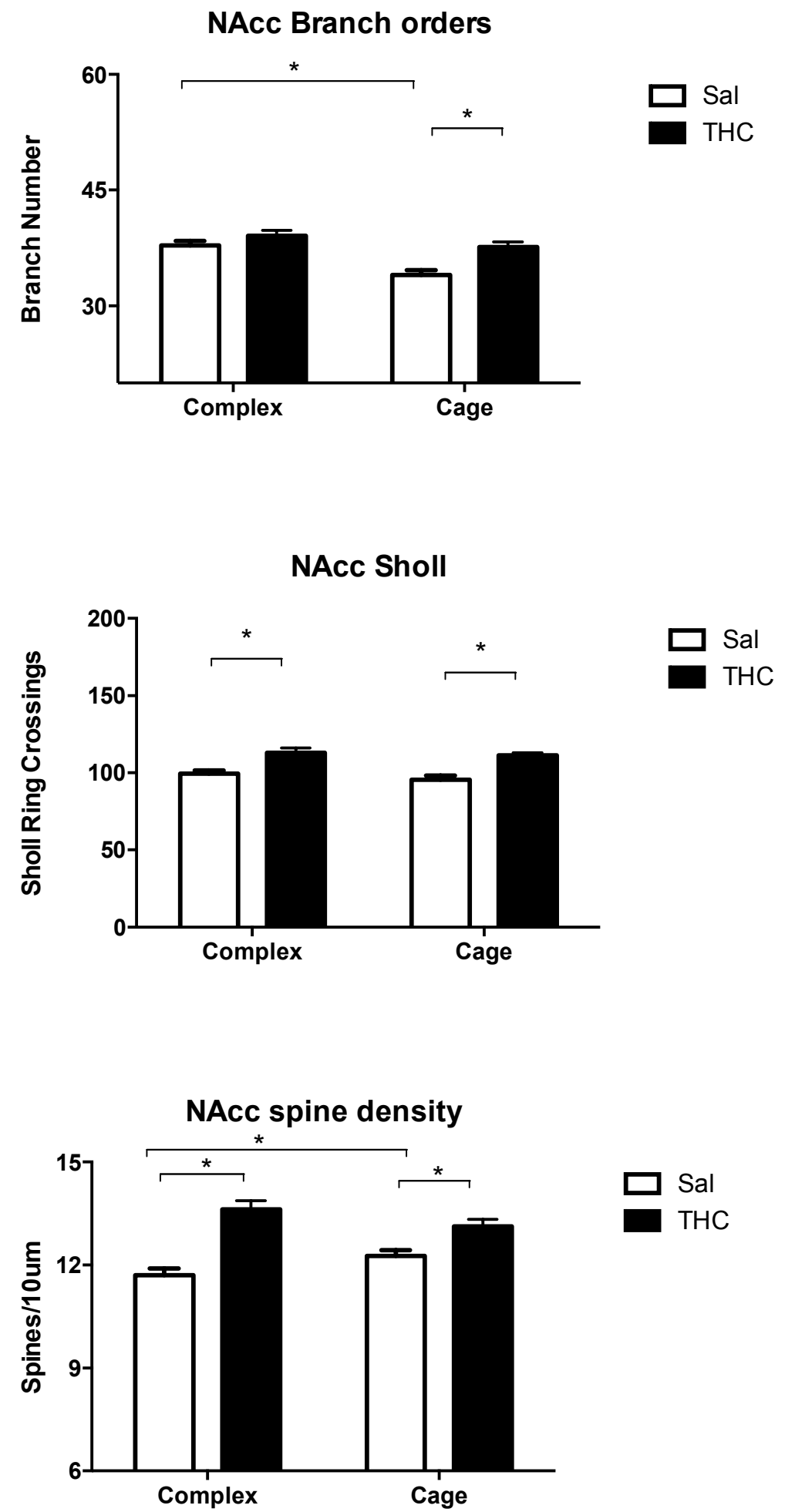
Both repeated exposure to Delta 9-Tetrahydrocannabinol (THC) and housing in complex environments changes the morphology of dendrites in medial prefrontal cortex, orbital frontal cortex and nucleus accumbens. Prior exposure to THC influenced the effect of later experience in complex environments to shape the structure of neurons in these three regions.

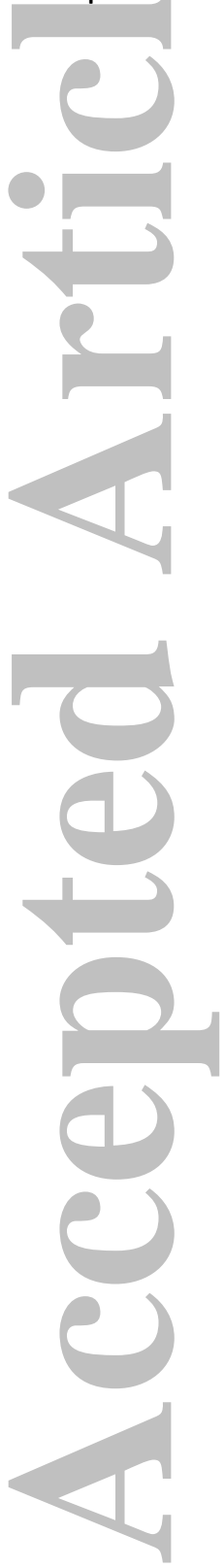




\section{Cg3 spine density}
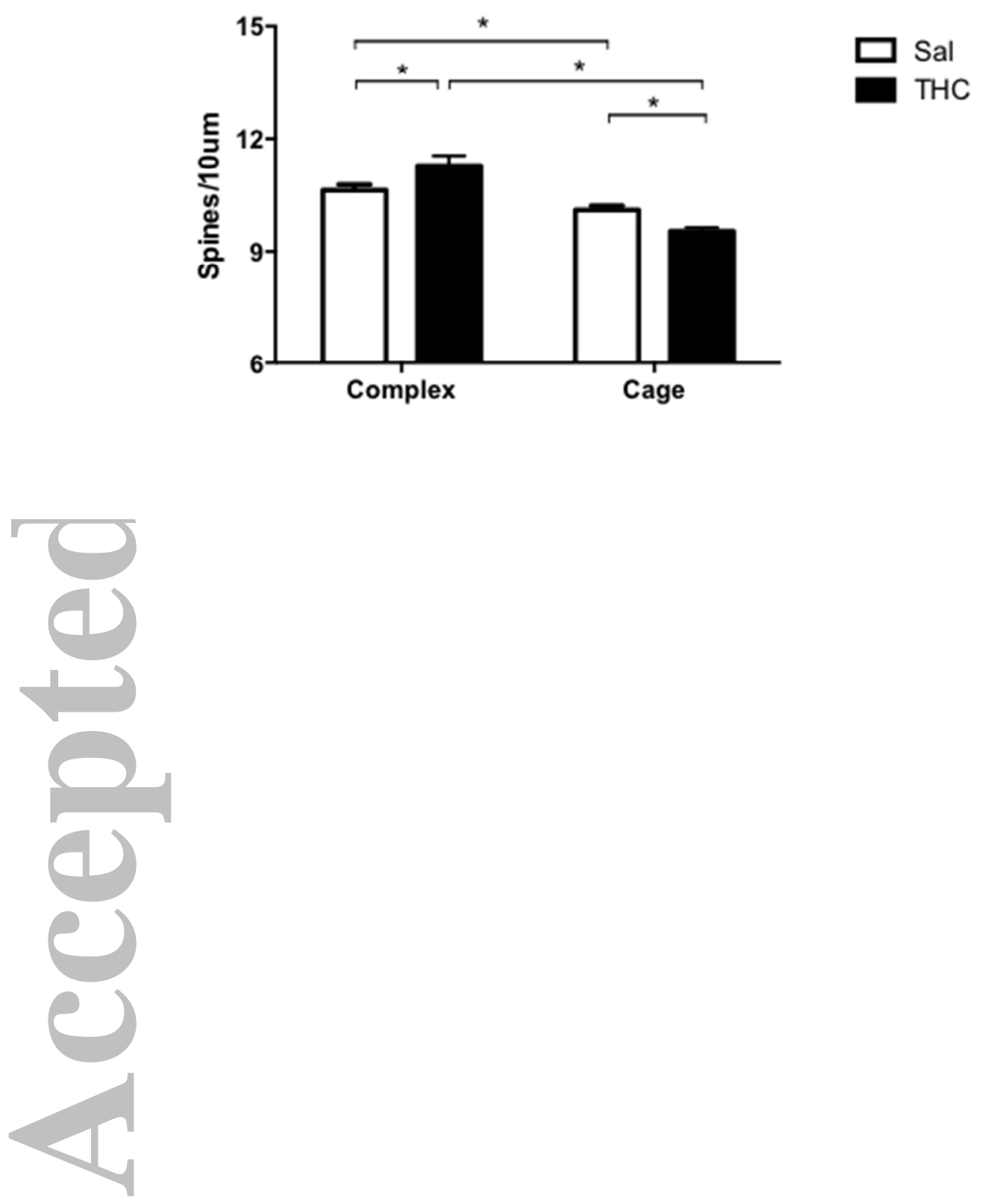